\title{
Oscillatory migratory large-scale fields in mean-field and direct simulations
}

\author{
Dhrubaditya Mitra ${ }^{1}$, Reza Tavakol ${ }^{1}$, Axel Brandenburg ${ }^{2,3}$ \\ and Petri J. Käpylä ${ }^{2,4}$ \\ ${ }^{1}$ Astronomy Unit, School of Mathematical Sciences, Queen Mary University of London, \\ Mile End Road, London E1 4NS, UK \\ emails: dhruba.mitra@gmail.com; r.tavakol@qmul.ac.uk \\ ${ }^{2}$ NORDITA, Roslagstullsbacken 23, SE-10691 Stockholm, Sweden \\ email: brandenb@nordita.org, \\ ${ }^{3}$ Department of Astronomy, Stockholm University, SE 10691 Stockholm, Sweden, \\ ${ }^{4}$ Observatory, Tähtitorninmäki (PO Box 14), FI-00014, University of Helsinki, Finland \\ email: petri.kapyla@helsinki.fi
}

\begin{abstract}
We summarise recent results form direct numerical simulations of both non-rotating helically forced and rotating convection driven MHD equations in spherical wedge-shape domains. In the former, using perfect-conductor boundary conditions along the latitudinal boundaries we observe oscillations, polarity reversals and equatorward migration of the large-scale magnetic fields. In the latter we obtain angular velocity with cylindrical contours and largescale magnetic field which shows oscillations, polarity reversals but poleward migration. The occurrence of these behviours in direct numerical simulations is clearly of interest. However the present models as they stand are not directly applicable to the solar dynamo problem. Nevertheless, they provide general insights into the operation of turbulent dynamos.
\end{abstract}

Keywords. MHD, convection, Sun: magnetic fields

\section{Introduction}

One of the major challenges in astrophysics is to understand the generation of largescale magnetic fields and their range of variations in a variety of settings, including the Sun, stars and galaxies. An important shared feature of such variations is that they often have characteristic time scales that are much shorter than the corresponding microscopic diffusion times. This would require the presence of relatively rapid mechanisms both for generation and dissipation of large-scale magnetic fields. An important candidate for the generation of such fields is a turbulent magnetohydrodynamic dynamo.

Here we shall concentrate on three such variations, namely oscillations, migrationespecially equatorward migration as observed for example in the Sun-and the approximately periodic reversal of field polarity.

A great deal of effort has gone into the study of these types of behaviour in dynamo models over the last few decades. These studies have employed both the mean-field (MF) approach (see e.g. Krause \& Rädler 1980; Moffatt 1978; Ossendrijver 2003) as well as direct numerical simulations (DNS) (see e.g. Brandenburg \& Subramanian 2005, and references therein). In the simplest MF approach the effects of turbulence are parametrised in terms of the turbulent magnetic diffusion and an $\alpha$ effect.

MF models can be classified according to the relative magnitudes of $\alpha$ effect and differential rotation $|\nabla \Omega|$, where $\Omega$ is the angular velocity in the convection zone. In general the $\alpha$ effect contributes to the generation of both poloidal and toroidal field components (the $\alpha^{2} \Omega$ dynamo). Often the $\alpha \Omega$ approximation (appropriate when the differential rotation 
is relatively large) and $\alpha^{2}$ models (which are appropriate when the differential rotation is negligible) are used. It is well known that $\alpha \Omega$ models possess oscillating spatiotemporal (wave-like) solutions (Parker 1955; Krause \& Rädler 1980). These oscillations propagate equatorward when the product $\alpha \frac{\partial \Omega}{\partial r}$ is negative in the northern hemisphere (Parker 1955; Yoshimura 1975) (see also Choudhuri 1998, for a recent account).

Mean field models have been phenomenologically successful in accounting for a number of features seen in the Sun and stars (see e.g. Ossendrijver 2003; Charbonneau 2005). For example, nonlinear $\alpha^{2} \Omega$ MF models which include the back reaction of Lorentz force on the azimuthal flow have been shown to be capable of producing many important features of the spatiotemporal variations observed in the Sun, including the torsional oscillations that penetrate the convection zone, as indicated by recent helioseismological inversions (see e.g. Covas et al. 2000a,b, 2004). Similar MF models have also been successfully used in order to account for a number of observed features in late-type rapidly rotating stars (Covas et al. 2005). So far, however, these successes have relied on rather ad hoc assumptions. For example to obtain the correct equatorward migration in the case of the Sun, the sign of $\alpha$ needs to be taken to be negative in the northern hemisphere. This follows from Parker's result (Parker 1955) linking the direction of migration with the sign of $\alpha \partial \Omega / \partial r$, and conflicts with contemporary results for the sign of $\alpha$ in the bulk of the convection zone in each hemisphere (Yoshimura 1975).

Other ways of producing the correct sense of migration in the Sun includes the incorporation of a meridional circulation (see e.g. Choudhuri et al. 1995) by assuming that the $\Omega$ effect operates at the bottom of the convection zone in order to generate a toroidal field, which in turn gives rise to a poloidal field by an $\alpha$ effect operating near the surface, and these are coupled by a meridional circulation which is assumed to have a poleward direction near the solar surface and an equatorward direction at the base of the convection zone. This circulation, which has not been directly observed in helioseismological inversions except near the surface, would need to be strong enough to make the equatorward migration possible. It is fair to say that despite their successes, all MF models require a number of ad hoc assumptions and choice of values of solar/stellar parameters that are only known very approximately at present (see e.g. Jouve et al. 2008; Chatterjee et al. 2004) for a list of such models.

The above shortcomings (as well as other fundamental questions such as the debate regarding the 'catastrophic quenching' of nonlinear dynamos) and the need to test various assumptions made in MF models, have in parallel motivated the study of turbulent dynamos using direct numerical simulations. Despite much effort and a number of important successes, however, direct numerical simulations of the full MHD equations in spherical shells have so far had limited success in generating large-scale magnetic fields on dynamical time scales (Gilman 1983; Glatzmaier 1985; Brown et al. 2009).

In the following we summarise some of our recent results, concerning oscillations, migrations of large-scale magnetic fields and polarity reversals, obtained using DNS in (i) helically forced MHD (Mitra et al. 2009) and (ii) convection-driven dynamos in a rotating frame (Käpylä et al. 2009).

\section{Oscillation and migration of large-scale fields in DNS}

In contrast to the MF studies, direct numerical simulations of turbulent MHD equations have seldom shown large-scale oscillations and migration. Two noteworthy exceptions are Brandenburg et al. (2001) and Gilman (1983). The former is a DNS with forced turbulence in Cartesian domains with imposed shear which gave rise to dynamo waves on 
time scales determined by the turbulent magnetic diffusivity (as opposed to the molecular diffusivity, which would imply that the oscillations were on dissipative time scales). The latter is a convection simulation in a spherical wedge-shaped domain, which showed poleward migration of magnetic activity-as opposed to equatorward migration seen in the Sun-and cylindrical contours of mean angular velocity-as opposed to cone-shaped contours in most of the solar convection zone. In the following we briefly review two recent migratory oscillating solutions obtained by DNS of the MHD equations (see Mitra et al. 2009; Käpylä et al. 2009, for details). In these studies we perform simulations in a spherical wedge domain as a compromise between requirements of high resolution and globality. We choose our domain to be distributed symmetrically about the equator.

\subsection{Forced DNS of the MHD equations}

We first describe the results of direct numerical simulations of the MHD equations with external forcing in a spherical wedge-shaped domain. The external force is random and white-in-time and injects negative helicity in the northern hemisphere and positive in the southern. We do not explicitly include convection, stratification and rotation, which are known to be present in solar and stellar convection zones, but the helical forcing used here does implicitly model these features (Krause \& Rädler 1980). We use perfectconductor boundary conditions (i.e. set the normal component of the magnetic field to be zero on the boundaries) on all boundaries except the boundary with constant azimuth where we impose periodic boundary conditions. The large-scale magnetic field is defined by averaging over the azimuthal and radial directions. Figure 1a depicts a 'butterfly diagram', showing oscillation, polarity reversals and equatorward migration of large-scale magnetic field.

Further investigations have shown that these results are well reproduced by a MF model of an $\alpha^{2}$ dynamo where the sign of $\alpha$ is positive in the northern hemisphere and negative in the southern (see Mitra et al. 2009, for details). The characteristic time scales of oscillations are well approximated by $T_{\text {cyc }}=1 / \eta_{\mathrm{T}} k_{\theta}^{2}$ with $\eta_{\mathrm{T}}=(1 / 3) u_{\mathrm{rms}} / k_{\mathrm{f}}$, $k_{\mathrm{f}}=W_{\mathrm{rms}} / U_{\mathrm{rms}}, W=\nabla \times \mathbf{U}, k_{\theta}=2 \pi / L_{\theta}, L_{\theta}=R_{\odot} \Delta \theta$ where $\Delta \theta$ is the range of colatitude $(\theta)$ in our wedge-shaped domain and $R_{\odot}=1$ in our simulations. Furthermore our MF studies have shown that the ratio between the maximum amplitude of the largescale magnetic field and the equipartition magnetic field $\left(B_{\text {eq }}\right)$ decreases as a function of the magnetic Reynolds number, $\operatorname{Re}_{\mathrm{M}}=u_{\mathrm{rms}} / \eta k_{\mathrm{f}}$ as $\operatorname{Re}_{\mathrm{M}}^{-0.4}$. This is related to the well known phenomenon of catastrophic quenching. This catastrophic quenching is expected to be alleviated by an exchange of magnetic helicity across the equator. Such a helicity flux, which is thought to be mediated by diffusion, has not yet been verified in DNS. Brandenburg et al. (2009) recently incorporated such a flux in a MF model and have shown that at high enough magnetic Reynolds numbers the diffusive flux is able to alleviate the quenching. We refer the reader to Mitra et al. (2009) for details of the model, methods of simulations and elaboration of the results reported above.

Finally we note that oscillations in one or two dimensional models of $\alpha^{2}$ dynamos, where the sign of $\alpha$ changes within the domain are well known literature (Baryshnikova \& Shukurov 1987; Rädler \& Bräeuer 1987; Stefani \& Gerbeth 2005; Rüdiger \& Hollerbach 2004), but migrational properties have not been investigated earlier.

\subsection{Rotating, convective DNS in spherical wedge-shaped domain}

Next we summarise results obtained using simulations of convective dynamos in spherical wedge-shaped domains, that include rotation explicitly. We consider a computational setup where the initial stratification in the region below $r=0.7 R_{\odot}$ is convectively stable, whereas the region above is unstable. The convection is driven self-consistently by 
a heat flux imposed at the lower boundary. The radii of the bottom of the domain, bottom of the convectively unstable layer, and the top of the domain are given by $\left(r_{1}, r_{2}, r_{3}\right)=(0.6,0.7,1) R_{\odot}$. We impose stress-free boundary conditions for the velocity and 'open' vertical field boundary condition (i.e. set the tangential component of magnetic field to zero at the boundary) at $r=R_{\odot}$. Starting from an uniformly rotating initial state, rotational shear is generated, and we again obtain large-scale magnetic fields which show oscillations, polarity reversals and migration, but the migration in this case is poleward, see Fig. 1b. This behaviour can be reproduced by an $\alpha \Omega$ dynamo. An accurate measurement of $\alpha$ is difficult in the present case because the test-field method has yet to be applied to this problem. However we use the approximation $\alpha \propto-\overline{\boldsymbol{\omega} \cdot \mathbf{u}}$, where $\boldsymbol{\omega}=\boldsymbol{W}--\overline{\mathbf{W}}, \mathbf{u}=\boldsymbol{U}--\overline{\mathbf{U}}, W=\boldsymbol{\nabla} \times \mathbf{U}$ and an overbar denotes averaging over the azimuthal direction. A contour plot of the product $\alpha \mathrm{d} \Omega / \mathrm{d} r$ is given in Fig. 1c. This shows that in clear agreement with the result of Parker (1955), that the sign of $\alpha \mathrm{d} \Omega / \mathrm{d} r$ determines the direction of migration. The details of the model, methods of simulation and elaboration of the results given in this Section can be found in (Käpylä et al. 2009).
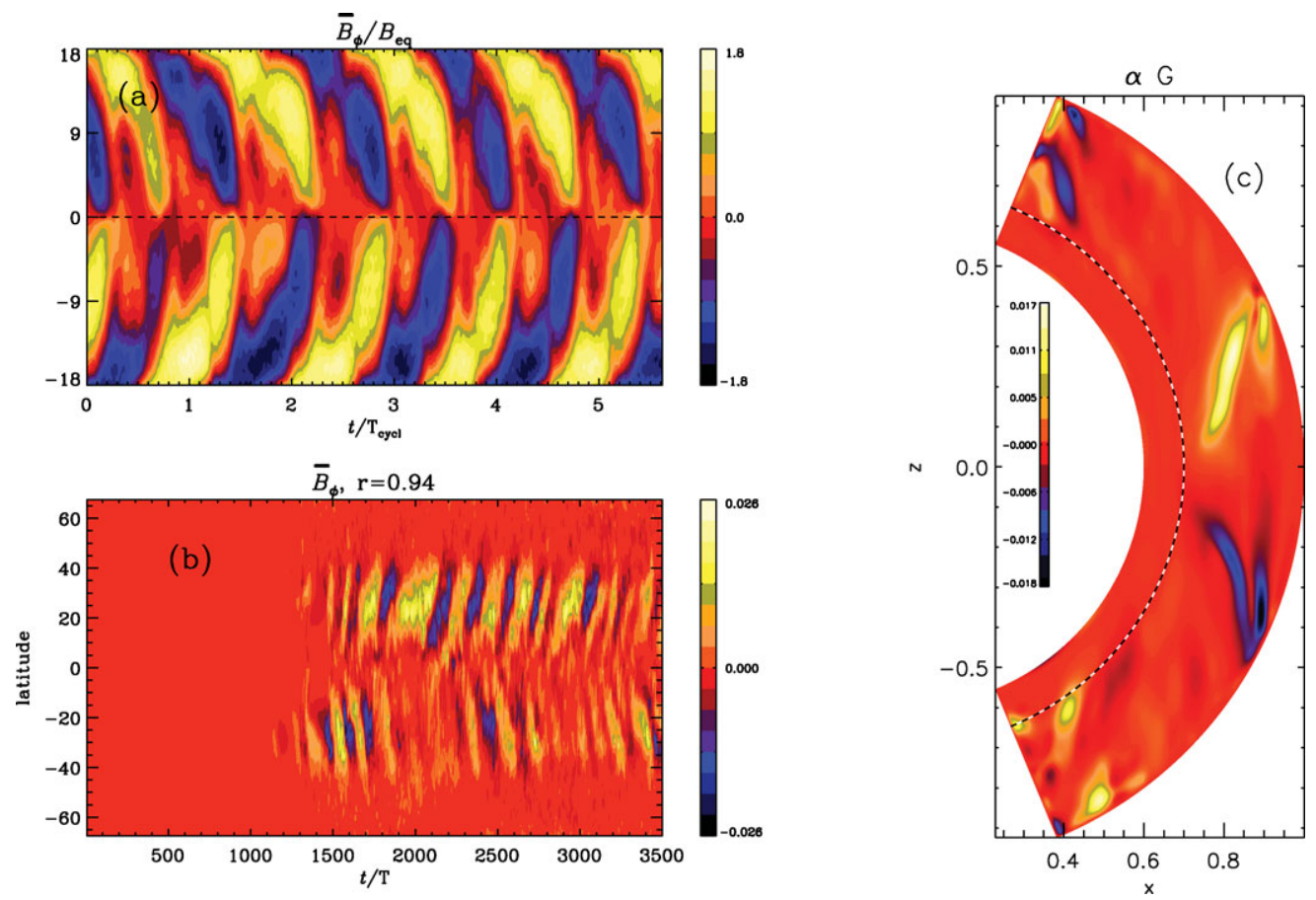

Figure 1. (a) Butterfly diagram for the toroidal field from the simulations of helically forced MHD equations in spherical wedge-shaped domain. (b) Butterfly diagram of the toroidal field from the simulations of rotating, convective MHD equations in spherical wedge-shaped domain. (c) A contour plot of a the product $\alpha G$ in the meridional plane from our convective simulations.

\section{Discussion}

Motivated by the observations of solar magnetic fields, we have considered oscillations, polarity reversals and migration of large-scale magnetic fields using direct numerical simulations We have summarised our recent results obtained using DNS of both nonrotating helically forced and rotating convection driven MHD equations in spherical wedge-shaped domains. 
In both cases we find large-scale magnetic fields are generated. In the non-rotating helically forced models, using perfect-conductor boundary conditions along the latitudinal boundaries, we find the field shows oscillations, polarity reversals and equatorward migration. In the case where convection and rotation are present we also find oscillations and polarity reversals, but the migration is in this case poleward.

Using analogous mean field models we have found that these results are well described by $\alpha^{2}$ and $\alpha \Omega$ mean field models respectively.

Clearly the occurrence of these features in direct numerical simulations is of interest. Nevertheless, the present models as they stand are not directly applicable to the solar dynamo problem, even though they provide insight into the operations of turbulent dynamos.

\section{Acknowledgement}

We would like to thank David Moss for very helpful discussions.

\section{References}

Baryshnikova, I. \& Shukurov, A. 1987, AN, 308, 89

Brandenburg, A. 2005, ApJ, 625, 539

Brandenburg, A., Bigazzi, A., \& Subramanian, K. 2001, MNRAS, 325, 685

Brandenburg, A., Candelaresi, S., \& Chatterjee, P. 2009, MNRAS, 398, 1414

Brandenburg, A., Rädler, K.-H., Rheinhardt, M., \& Subramanian, K. 2008, ApJ Lett., 687, L49

Brandenburg, A. \& Subramanian, K. 2005, Phys. Rep., 417, 1

Brown, B. P., Browning, M. K., Miesch, M. S., Brun, A. S., \& Toomre, J. 2009, ApJ (submitted), arXiv:0906.2407; see also, Brown, B. P., Browning, M. K., Brun, A. S., Miesch, M. S. et al. 2007, AIPC, 948, 271; Browning, M. K., Miesch, M. S., Brun, A. S., \& Toomre, J. 2006, ApJ, 648, L157; Brun, A. S., Miesch, M. S., \& Toomre, J. 2004, ApJ, 614, 1073

Charbonneau, P. 2005, Living Reviews in Solar Physics, 2

Chatterjee, P., Nandy D., \& Choudhuri, A. 2004, A\&SA, 427, 1019

Covas, E., Tavakol, R., Moss, D., \& Tworkowski, A. 2000, A\& A, 360, L21

Covas, E., Tavakol, R., \& Moss, D. 2000, A\&GA, 363, L13

Covas, E., Moss, D., \& Tavakol, R. 2004, A\&A, 416, 775

Covas, E., Moss, D., \& Tavakol, R. 2005 A $\& A$, 429, 657

Choudhuri, A. 1998, The physics of Fluids and Plasmas: An introduction for Astrophysicists. Cambridge University Press, Cambridge

Choudhuri, A. R., Schussler, M., \& Dikpati, M. 1995, A\&AA, 303, L29+

Gilman, P. A. 1983, ApJS, 53, 243

Glatzmaier, G. A. 1985, ApJ, 291, 300

Jouve, L., Brun, A. S., Arlt, R., Brandenburg, A., Dikpati, M., Bonanno, A., Käpylä, P. J., Moss, D., Rempel, M., Gilman, P., Korpi, M. J., \& Kosovichev, A. G. 2008, A\& $\mathcal{A}$ A, 483, 949

Käpylä, P. J., Korpi, M. J., Brandenburg, A., Mitra, D., \& Tavakol, R. 2009, arXiv:0909.1330

Krause F. \& Rädler, K.-H. 1980, Mean-field magnetohydrodynamics and dynamo theory. Pergamon Press, Oxford

Mitra, D., Tavakol, R., Käpylä, P. J., \& Brandenburg, A. 2009, arXiv:0901.2364

Moffatt, K. 1978, Magnetic Field Generation in Electrically Conducting Fluids. Cambridge University Press, Cambridge.

Ossendrijver, M. 2003, Astron. Astrophys. Rev, 11, 287-367 (2003).

Parker, E. 1955, ApJ, 1122, 293

Parker, E. 1987, Sol. Phys., 110, 11

Rädler, K.-H. \& Bräeuer, H.-J. 1987, AN, 308, 101

Rüdiger, G. \& Hollerbach, R. 2004, in Rüdiger, G., Hollerbach, R., eds, The Magnetic Universe: Geophysical and Astrophysical Dynamo Theory. Wiley-VCH, p. 343

Stefani, F. \& Gerbeth, G. 2005, Phys. Rev. Lett, 94, 184506

Yoshimura, H. 1975, ApJ. 201, 740. 